\title{
Lower bound for average delay in unblocked random access algorithm with orthogonal preambles
}

\author{
A. A. Burkova, Assistant Professor, orcid.org/0000-0002-0920-585X \\ S. V. Shneerb', PhD, Phys.-Math., Associate Professor, orcid.org/0000-0001-6750-6995 \\ A. M. Turlikova , Dr. Sc., Tech., Professor, orcid.org/0000-0001-7132-094X, turlikov@k36.org \\ aSaint-Petersburg State University of Aerospace Instrumentation, 67, B. Morskaia St., 190000, \\ Saint-Petersburg, Russian Federation \\ bSchool of Mathematical and Computer Sciences, Heriot-Watt University, Edinburgh, EH14 4AS, \\ United Kingdom
}

\begin{abstract}
Introduction: Currently, the first versions of $5 \mathrm{G}$ communication standard networks are being deployed and discussions are underway on further development of cellular networks and transition to the $6 \mathrm{G}$ standard. Once the Internet of Things is fully developed, it will function within the framework of Massive Machine-Type Communications. Its performance will need to satisfy a number of requirements: very high energy efficiency, relatively low delay and fairly reliable communication. Due to the nature of the traffic, it is impossible to develop a channel resource sharing policy and thus random multiple access procedures will be employed. A class of unblocked algorithms with orthogonal preambles may be used to increase the efficiency of random access. Purpose: To calculate the lower bound of the average delay for the class of unblocked random multiple access algorithms using orthogonal preambles. Results: We propose a model of a system with a potentially unlimited number of users who utilize random unblocked access to transmit data over a common communication channel using orthogonal preambles. For an infinite number of preambles, we prove that up to an input arrival rate of 0.5671 , the system is stable. We obtain a closed-form expression for the average delay in a stable system depending on the intensity of the input arrival rate. We also demonstrate that this expression is the lower bound for the average delay in a system with a finite number of preambles. We perform a simulation in the case of a finite number of preambles which shows that with an increase in the number of preambles, the input arrival rate at which the system is stable approaches 0.5671 , and the average delay tends to the lower bound. With an input arrival rate not exceeding 0.3 , about 100 orthogonal preambles are sufficient to reach the lower bound. Practical relevance: The obtained bound allows us to estimate the lower average delay in the class of algorithms under consideration. Its application allows us to determine the possibility of using such a class of algorithms at the stage of designing random multiple access systems from the point of view of limitations on the average delay.
\end{abstract}

Keywords - random multiple access, maximum arrival rate, average delay, preambles, mMTC, IoT.

For citation: Burkov A. A., Shneer S. V., Turlikov A. M. Lower bound for average delay in unblocked random access algorithm with orthogonal preambles. Informatsionno-upravliaiushchie sistemy [Information and Control Systems], 2020, no. 3, pp. 79-85. doi:10.31799/1684-8853-2020-3-79-85

\section{Introduction}

Currently, the first versions of $5 \mathrm{G}$ communication standard networks are being deployed and new versions will be developed in the near future [1-3]. Within the framework of the fifth generation networks, three main types of communication will be distinguished: enhanced Mobile Broadband, UltraReliable Low Latency Communication, and Massive Machine-Type Communications (mMTC) [4]. In further development of cellular networks, and the transition to the $6 \mathrm{G}$ standard, these key scenarios are expected to remain relevant, but the level of requirements for the main characteristics will increase $[1,3]$. The functionality of the currently popular idea of the Internet of Things (IoT) is expected to be within the framework of the mMTC scenario $[3,5]$. For this type of communication there are a number of requirements for performance characteristics: very high energy efficiency, relatively low latency and fairly reliable communication [1]. The task of meeting these requirements is difficult, due to the features of IoT traffic. A huge number of devices generate data periodically and unpredictably. Due to the number of devices in the network and the characteristics of the formation of data traffic, it is difficult to develop an effective channel resource allocation policy $[4,5]$. Therefore, in the framework of this scenario, it is assumed that random multiple access procedures are used when devices access the shared resource uncoordinated, as well as without the need for a connection establishment and communication with the resource manager [5].

The 5G standard is advancing as the primary performance tool for IoT communications, thanks to the development of 5G New Radio. However, the revolutionary forecast for $5 \mathrm{G}$ networks was reduced exclusively to work on mmWave, and that would not allow for the operation of IoT systems taking into account the increasing number of devices and services [5]. When applying mmWave communication to cellular systems, an important problem that can negate its advantages is multiple access, namely because of the high energy consumption and high cost 
of equipment [6]. However, this is only the first prerequisite for the development of IoT technology and future versions of cellular standards will require new solutions in this direction.

In this paper, we are going to consider the use of orthogonal preambles for data transmission in mMTC scenarios. A similar approach to data transmission using orthogonal preambles is considered in [7]. The algorithm considered there involves work in two stages. At the first stage, users transmit preambles, then they receive information about which slot the user who sent a particular preamble will utilize. The base station then allocates preamble resources for data transmissions. At the second stage, the user transmits data to the allocated time-frequency resource in accordance with the transmitted preamble and the received information. In [7], the authors note that with a limited number of preambles, the system can quickly become unstable, therefore, it considers a variant of the lossy algorithm. That is, if several users transmit under the preamble in the allocated resource, then the message is lost. We note further the following features. When working with losses, the system is always stable. When data is divided into two types and the operation of the system into two distinct stages, the user has to constantly monitor the channel output and the stages in the system, even if he does not have a message to transmit. At the command of the message, the user will have to wait for the next first stage of the system, postponing his transfer. This algorithm can be attributed to the class of blocked algorithms. The term blocked and non-blocked algorithms was first introduced in the works of B. S. Tsybakov (see, for example, [8]). The features of these types of algorithms are also discussed in $[9,10]$. A blocked algorithm runs in sessions. The user can transmit only in the first slot of the session, if the user appeared in the system during the session; then the user blocks his transmission until the start of the next session. Therefore, this algorithm is called blocked. An algorithm related to this type in the framework of the mMTC scenario was also considered in [11], in which, unlike in [7], there is no separation of messages into two types and no losses of messages. One of the disadvantages of this class of algorithms is the constant listening to the channel, even in the absence of a message ready for transmission by the user. As one of mMTC traffic characteristics is a rare message from the user, the constant monitoring of the channel adversely affects the energy efficiency of the system. For transmitting devices, with a limitation on complexity and energy efficiency, a significant role is played by delays between the appearance of a message from a user and its transmission to a communication channel. Therefore, more efficient algorithms are those in which, when a user has a message, it is transmitted in the next slot without any wait. Algorithms with this property form a class of non-blocked algorithms. With this approach, the user will listen to the channel only after the first transmission of the message that appears with him and until it is transmitted, and not continuously, as in blocked algorithms.

In this paper, we will consider an unblocked random multiple access algorithm using orthogonal preambles. The data transfer mode can be divided into two types. The first type is the first transmission of a message that occurs in a nearby slot. In the event of a collision, the preamble information will be used by the base station to schedule the time-sharing mode. The second type, in the event of a collision during the first transmission, is the message that is resent in accordance with the schedule received from the base station. For this algorithm, assuming an infinite number of preambles, we obtain a lower bound for the average number of users, the delay and the maximum input arrival rate at which the system is stable. We will also demonstrate the performance of the algorithm with a finite number of preambles for some implementation of the system, with lossless operation and a different number of preambles. We will further provide a comparison of the simulation results and the obtained lower bound.

\section{System model}

We will make a number of assumptions on the operation of the algorithm which will facilitate our analysis of the system characteristics.

Assumption 1. Messages for all users have the same length. The transmission time of one message is taken as a unit of time. The entire transmission time in the channel is divided into slots that correspond to the transmission time of one message. User messages consist of the service part (randomly selected preamble from some set) and data. Users know exactly the moments of breaking into slots and can transmit only at the beginning of the next slot (synchronous system).

Assumption 2. There are three possible events in the slot: slot;

"Empty" $(E)$, if no one was transmitting in the

"Success" $(S)$, if one user transmitted in the slot. In this case, the base station correctly receives both the preamble and the message itself;

"Conflict" $(C)$, if more than one user was transmitted in one slot. That message cannot be correctly received by the base station. Preamble numbers are accepted correctly, even if several users choose one preamble. 
Assumption 3. The base station transmits information about the event that occurred in the channel and additional information necessary for the algorithm to work (for example, a list of preambles involved in the conflict).

Assumption 4. An infinite number of users is assumed. The number of new messages that appear in the system in one slot is distributed according to the Poisson law with the parameter $\lambda$, where is the intensity of the input arrival rate.

Assumption 5. There is an infinite number of orthogonal preambles.

According to assumption 4, each message appearing in the system will be considered a new user who leaves the system after the transmission of this message.

Algorithm for users.

1. The user, when it has a message, selects one of the preambles in a random fashion. And it transmits a message consisting of the selected preamble and data at the beginning of the next slot.

2 . The user receives information from the base station and acts in accordance with it:

"Success": the user deletes the message because it was sent successfully;

"Conflict": the user delays the transmission of its message to the slot allocated under its preamble in accordance with the schedule received from the base station.

The algorithm of the base station for an infinite number of preambles.

The base station receives information from the channel and operates depending on the events in the channel:

"Empty": the base station informs all users of the "Empty" event;

"Success": the base station informs all users about the "Success" event along with the number of the received preamble. If the system was in the time-sharing mode, the base station removes this preamble from the schedule;

"Conflict": the base station reports a "Conflict" along with the numbers of the preambles involved in it. The base station makes or adjusts the schedule based on the detected preambles and reports it to users.

An example of the algorithm operation is presented in Fig. 1. According to assumptions 1 and 5, all users appearing in the system select unique orthogonal preambles. Assume that there are 5 users $\left(U_{1}\right.$, $\left.U_{2}, U_{3}, U_{4}, U_{5}\right)$. At the beginning of the second slot, the first user $\left(U_{1}\right)$, which has a message, sends it to the channel and the event that occurs is "Success". In the second slot, messages appear for users $U_{2}$ and $U_{3}$, which are transmitted in the third slot and the event "Conflict" occurs. The base station correctly decodes the numbers of the preambles involved in the conflict and schedules the next two slots. The

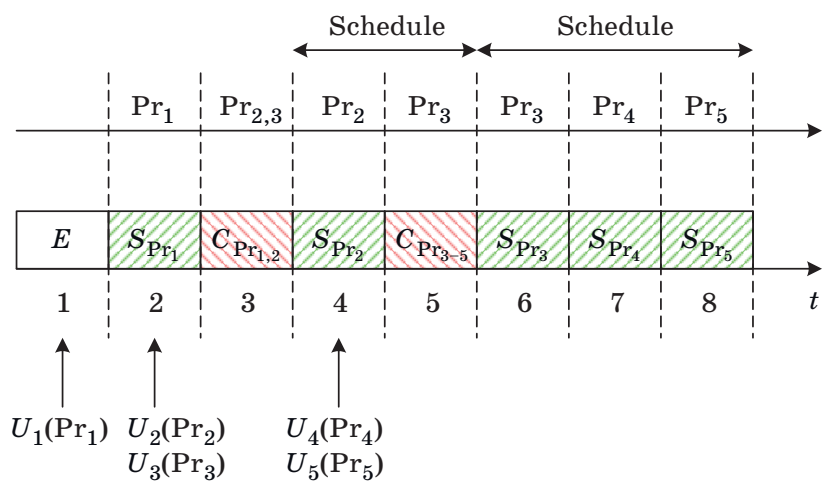

Fig. 1. An example of an unblocking random multiple access algorithm with orthogonal preambles

second user successfully sends the message according to the schedule. Users $U_{4}$ and $U_{5}$ appear in the fourth slot. In the fifth slot, they transmit messages, thereby causing a conflict and interrupting the current schedule. The base station decodes the numbers of the preambles involved in the conflict, adjusts the schedule, and informs its users. In the sixth through eighth slots, no new messages appear, and users $\left(U_{3-5}\right)$, successfully transmit their messages according to the schedule.

Remark 1. In real systems, there cannot be an infinite number of preambles, and assumption 5 is not valid for such systems. In the case of a limited number of preambles, it is necessary to additionally determine the rule of operation of the algorithm in the case when a "Conflict" occurs in one frequency-time resource, and several users choose the same preamble.

\section{System analysis}

In the system under consideration, messages can appear during the slot and affect the system in the next slot. So if the system is in the time-sharing mode, the message that appears during the slot will cause a conflict in the next slot. To take into account such events when describing the number of active users in the system using a recurrence equation, one needs to use a two-dimensional Markov chain (for example, [12]). The use of such a model makes an exact analysis of the system impossible. Therefore, for our analysis, we will use an auxiliary system in which messages can appear only at the beginning of the slot and therefore can be transmitted immediately. An example showing the difference between the basic and auxiliary systems is shown in Fig. 2.

By comparing the basic and auxiliary systems, one can prove the following statement.

Statement 1. Let $\bar{N}(\lambda)$ be the average number of users in the main system, and let $\bar{Q}(\lambda)$ be the aver- 


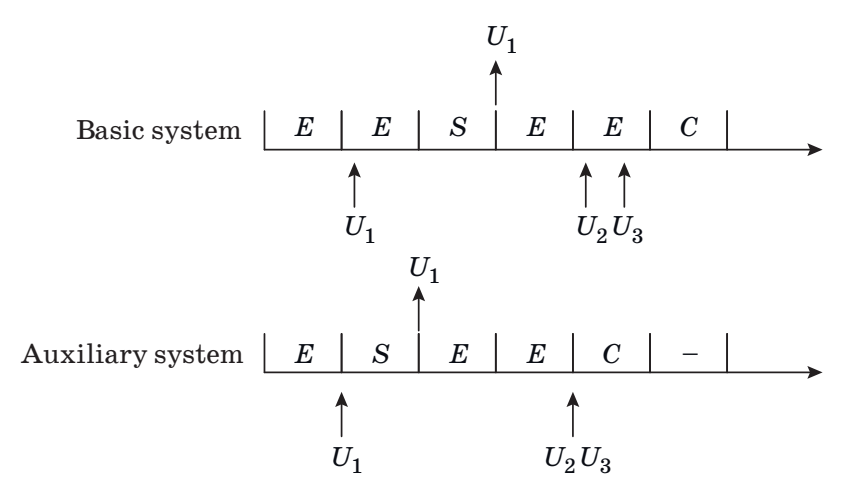

Fig. 2. The difference between the basic and auxiliary systems

age number of users in the auxiliary system, where $\lambda$ is the input stream intensity. The following equality holds:

$$
\bar{N}(\lambda)=\bar{Q}(\lambda)+\lambda
$$

We perform further calculations for the auxiliary system. Denote by $Q_{k}$ by the number of users in the slot with number $k$. Then the number of users in the next slot satisfies the following recursive equation describing the operation of the system:

$$
\begin{gathered}
Q_{k+1}=Q_{k}+V_{k}-I\left(V_{k}=1\right) \times \\
\times I\left(Q_{k}=0\right)-I\left(V_{k}=0\right) I\left(Q_{k}>0\right),
\end{gathered}
$$

where $V_{k}$ is the number of users appearing in the slot with number $k$, and $I$ (statement) is the indicator function, which takes values:

$$
I\{\text { statement }\}=\left\{\begin{array}{l}
1, \text { true } \\
0, \text { false }
\end{array} .\right.
$$

Using expression (2), one can easily analyze the stability of the system. Suppose that initially $Q_{0}=q$ where $q$ is a large value. Then

$$
\mathrm{E}\left[Q_{1}-Q_{0} \mid Q_{0}=q\right]=\lambda-e^{-\lambda} .
$$

Stability is ensured when the value of the expression (3) is negative (see [13]). This is equivalent to $\lambda<e^{-\lambda}$, a solution to this inequality can be found using the Lambert function (W) [14], and we obtain $\lambda<W(1) \approx 0.5671$.

We will assume that the system works indefinitely. If the system is stable, then $Q_{k}$ and $Q_{k+1}$ have the same distribution, and, in particular, the same moments. We take the mathematical expectation of the left and right sides of formula (2) and get

$$
\begin{gathered}
\mathrm{E}\left[Q_{k+1}\right]= \\
=\mathrm{E}\left[Q_{k}\right]+\lambda-\lambda e^{-\lambda} \pi_{0}-e^{-\lambda}\left(1-\pi_{0}\right),
\end{gathered}
$$

where by $\pi_{0}$ we denote the stationary probability that the system is empty. Using that $\mathrm{E}\left[Q_{k+1}\right]=\mathrm{E}\left[Q_{k}\right]$, we can write

$$
\lambda=\lambda e^{-\lambda} \pi_{0}+e^{-\lambda}\left(1-\pi_{0}\right),
$$

which implies

$$
\pi_{0}=\frac{e^{-\lambda}-\lambda}{e^{-\lambda}(1-\lambda)}
$$

Squaring the right- and the left-hand sides of formula (2) and taking the mathematical expectation, we obtain the following expression:

$$
\begin{gathered}
\mathrm{E}\left[\left(Q_{k+1}\right)^{2}\right]=\mathrm{E}\left[\left(Q_{k}\right)^{2}\right]+\left(\lambda^{2}+\lambda\right)+ \\
+\left(\lambda e^{-\lambda} \pi_{0}-e^{-\lambda}\left(1-\pi_{0}\right)\right)+ \\
+2 \mathrm{E}\left[Q_{k}\right] \lambda-2 \mathrm{E}\left[Q_{k}\right] e^{-\lambda}-2 \lambda e^{-\lambda} \pi_{0} .
\end{gathered}
$$

Using $\mathrm{E}\left[\left(Q_{k+1}\right)^{2}\right]=\mathrm{E}\left[\left(Q_{k}\right)^{2}\right]$ and using formula (6), we obtain

$$
0=\lambda^{2}+2 \lambda-2 \mathrm{E}\left[Q_{k}\right]\left(e^{-\lambda}-\lambda\right)-2 \lambda e^{-\lambda} \pi_{0} .
$$
tion

Next, using expression (5) we obtain the equa-

$$
2 \mathrm{E}\left[Q_{k}\right]\left(e^{-\lambda}-\lambda\right)=\lambda^{2}+2 \lambda-2 \lambda \frac{e^{-\lambda}-\lambda}{(1-\lambda)},
$$

which implies

$$
\begin{gathered}
\mathrm{E}\left[Q_{k}\right]=\frac{\lambda^{2}+2 \lambda-2 \lambda \frac{e^{-\lambda}-\lambda}{(1-\lambda)}}{2\left(e^{-\lambda}-\lambda\right)}= \\
=\frac{\lambda^{2}(1-\lambda)+2 \lambda\left(1-e^{-\lambda}\right)}{2\left(e^{-\lambda}-\lambda\right)(1-\lambda)} .
\end{gathered}
$$

Expression (7) determines the average number of users in the auxiliary system at $k \rightarrow \infty$, with a fixed $\lambda<0.5671$. Let us denote the right-hand side of expression (7) by $\bar{Q}(\lambda)$. Using statement 1 , the average number of users in the main system is defined as:

$$
\begin{aligned}
\bar{N}(\lambda) & =\frac{\lambda^{2}(1-\lambda)+2 \lambda\left(1-e^{-\lambda}\right)}{2\left(e^{-\lambda}-\lambda\right)(1-\lambda)}+\lambda= \\
& =\frac{2 \lambda-\lambda^{2}\left(1+2 e^{-\lambda}-\lambda\right)}{2\left(e^{-\lambda}-\lambda\right)(1-\lambda)} .
\end{aligned}
$$

Using Little's theorem [15] and expression (8), we can determine the average delay $\bar{D}$ in the main system as 


$$
\bar{D}=\frac{\mathrm{E}\left[N_{k}\right]}{\lambda}+0.5=\frac{2-\lambda\left(1+2 e^{-\lambda}-\lambda\right)}{2\left(e^{-\lambda}-\lambda\right)(1-\lambda)}+0.5
$$

Remark 2. The addition of 0.5 in formula (9) is determined by the fact that the synchronous system is considered (see assumption 1) and we thus need to take into account the average waiting time for the next slot.

\section{Numerical results}

In this section we present simulation results for the system under consideration for a different number of preambles. Taking into account Remark 1, for a finite number of preambles, we modify the algorithm as follows. If a user finds itself in a conflict situation and at the same time the base station detects only one preamble, then the users participating in such a conflict will retransmit their message with probability $p=\min \left(\frac{L}{N_{a}}, 1\right)$, where $L$ is the number of orthogonal preambles and $N_{a}$ is the number of users who have a message ready for transmission at the time of the start another slot. It is worth noting that in real systems, knowledge of the value $N_{a}$ may not be available, but there are works $[16,17]$ in which methods for its evaluation are proposed. Such a modification of the algorithm for a finite number of preambles is logical, but it may not achieve the best performance. However, its influence on the performance of the system decreases with an increase in the number of preambles.

Figure 3 illustrates the average delay in the system for different values of the number of pre-

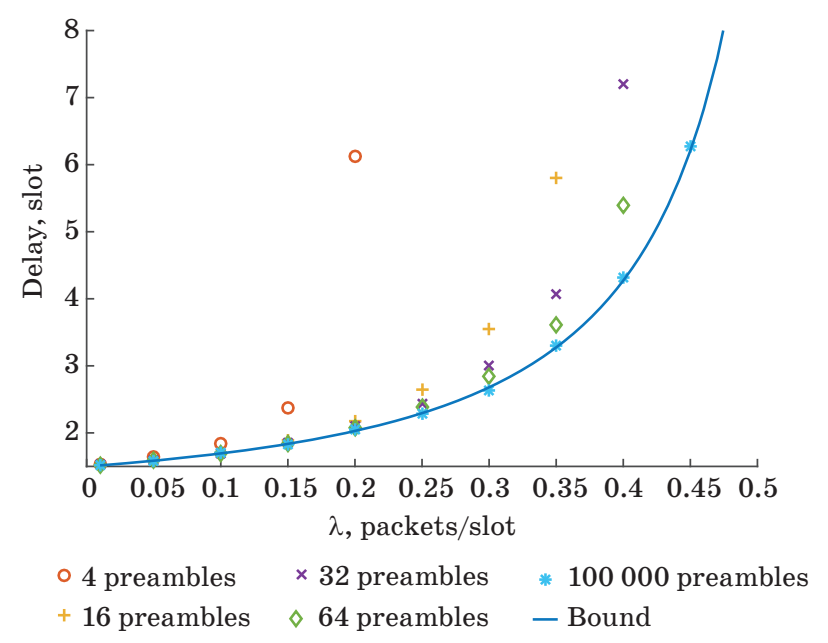

Fig. 3. The dependence of the average delay on the intensity of the input arrival rate for a different number of preambles

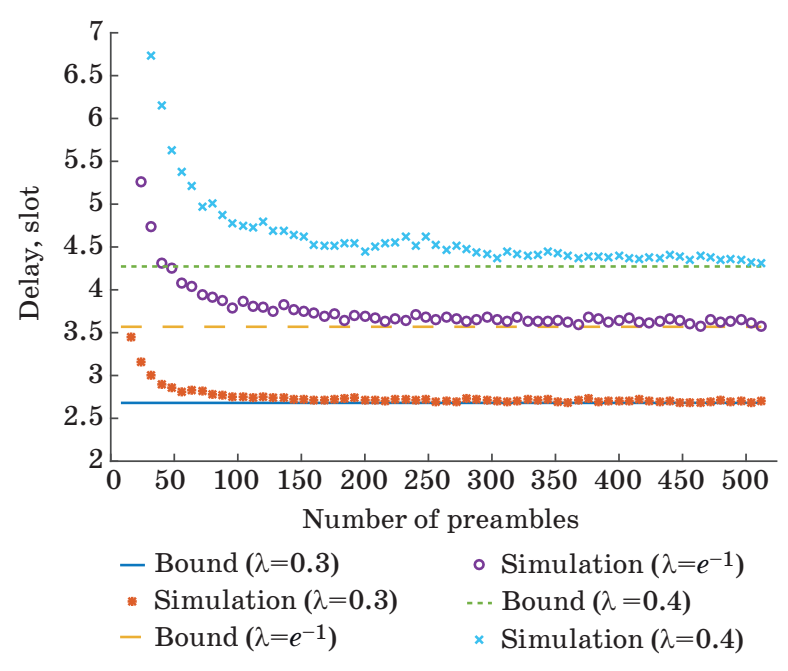

- Fig. 4. Values of an average delay from the number of preambles for input arrival rate equal to $0.3, e^{-1}$ and 0.4

ambles. The bound calculated by formula (9) is also shown. One can be see from the graph that with a small number of preambles, the delay values go to infinity earlier than the maximum input arrival rate obtained previously $(0.5671)$ at which the system is stable. This is due to the fact that with a finite number of preambles this intensity is lower and tends to the obtained value with an increase in the number of preambles. One can also see that with an increase in the number of preambles, the average delay values approach the lower bound.

Figure 4 shows the dependence of the average delay in the system for fixed values of the input arrival rate $\left(0.3, e^{-1}\right.$ and 0.4$)$ on the number of orthogonal preambles. For instance, for an input stream intensity of 0.3 , about 100 preambles are enough to reach the obtained bound.

\section{Conclusion}

We considered an unblocked random access algorithm using orthogonal preambles. When a message appears, users immediately send it. Messages consist of data and orthogonal preambles. The base station uses information about orthogonal preambles in the event of a conflict to construct a schedule that is reported to users. We introduced an auxiliary system under the assumption of an infinite number of preambles and carried out its rigorous analysis. We showed that the system with a large number of preambles is stable for arrival intensities up to the value $\lambda=0.5671$. We also obtained lower bounds for the average number of users and the average delay in the system. We further presented results of modeling with a finite number of preambles with some additional modification of the algorithm. A simulation has shown that with an increase in 
the number of preambles, the maximum intensity of the input arrival rate approaches the calculated one, and the delay approaches the obtained bound. The results of our simulation showed that at an input arrival rate of 0.3 , about 100 orthogonal preambles are sufficient to reach the lower bound. When using a finite number of preambles, there are various ways of implementing the algorithm in case of conflicts with the intersection of preambles for

\section{References}

1. Letaief K. B., Chen W., Shi Y., Zhang J., \& Zhang Y. J. A. The roadmap to 6G: AI empowered wireless networks. IEEE Communications Magazine, 2019, vol. 57, no. 8, pp. 84-90. doi:10.1109/MCOM.2019.1900271

2. Piran M. J., Suh D. Y. Learning-Driven Wireless Communications, towards 6G. 2019 International Conference on Computing, Electronics \& Communications Engineering (iCCECE), IEEE, 2019, pp. 219224. doi:10.1109/iCCECE46942.2019.8941882

3. Mahmood N. H., Alves H., López O. A., Shehab M., Osorio D. P. M., \& Latva-aho M. Six key enablers for machine type communication in 6G. arXiv preprint arXiv:1903.05406, 2019.

4. Popovski P., Trillingsgaard K. F., Simeone O., \& Durisi G. 5G wireless network slicing for eMBB, URLLC, and mMTC: A communication-theoretic view. IEEE Access, 2018, vol. 6, pp. 55765-55779. doi:10.1109/ACCESS.2018.2872781

5. Clazzer F., Munari A., Liva G., Lazaro F., Stefanovic C., \& Popovski P. From 5G to 6G: Has the time for modern random access come? arXiv preprint arX$i v: 1903.03063,2019$.

6. Zhu L., Xiao Z., Xia X. G., \& Wu D. O. Millimeter-wave communications with non-orthogonal multiple access for B5G/6G. IEEE Access, 2019, vol. 7, pp. 116123116132. doi:10.1109/ACCESS.2019.2935169

7. Choi J. On throughput of compressive random access for one short message delivery in IoT. IEEE Internet of Things Journal, 2020, vol. 7, no. 4, pp. 3499-3508. doi:10.1109/JIOT.2020.2972519

8. Tsybakov B. Survey of USSR contributions to random multiple-access communications. IEEE Transactions on Information Theory, 1985, vol. 31, no. 2, pp. 143165. doi:10.1109/TIT.1985.1057023 different users. A logical continuation of the work is the search for an algorithm that provides an average delay closest to the received lower bound.

\section{Acknowledgment}

The research of A. Burkov was supported by RFBR, project number 19-31-27001.

9. Rom R., Sidi M. Multiple Access Protocols: Performance and Analysis. Springer Science \& Business Media, 2012. 178 p. doi:10.1007/978-1-4612-3402-9

10. Chlebus B. S. Randomized Communication in Radio Networks. arXiv preprint arXiv:1801.00074, 2017.

11. Burkov A., Frolov A., Turlikov A. Contention-based protocol with time division collision resolution. 2018 10th International Congress on Ultra Modern Telecommunications and Control Systems and Workshops (ICUMT), IEEE, 2018, pp. 1-4. doi:10.1109/ ICUMT.2018.8631217

12. Burkov A. A., Saveliev A. A., Turlikov A. M. Upper and lower bound for non-blocking random multiple access algorithm with time division mode. 2019 Wave Electronics and its Application in Information and Telecommunication Systems (WECONF), IEEE, 2019, pp. 1-7. doi:10.1109/WECONF.2019.8840590

13. Meyn S. P., Tweedie R. L. Markov Chains and Stochastic Stability. Springer Science \& Business Media, 2012. 559 p. doi:10.1007/978-1-4471-3267-7

14. Corless R. M., Gonnet G. H., Hare D. E., Jeffrey D. J., \& Knuth D. E. On the Lambert W Function. Advances in Computational Mathematics, 1996, vol. 5, pp. 329 359. doi:10.1007/BF02124750

15. Brémaud P. Probability Theory and Stochastic Processes. Springer, Cham, 2020. 717 p. doi:10.1007/9783-030-40183-2

16. Jeon S. W., Jin H. Online Estimation and Adaptation for Random Access with Successive Interference Cancellation. arXiv preprint arXiv:2001.03248, 2020.

17. Galinina O., Turlikov A., Andreev S., \& Koucheryavy Y. Stabilizing multi-channel slotted aloha for machine-type communications. 2013 IEEE International Symposium on Information Theory, IEEE, 2013, pp. 2119-2123. doi:10.1109/ISIT.2013.6620600 
УДК 004.728.3.057.4

doi:10.31799/1684-8853-2020-3-79-85

Нижняя граница для средней задержки в неблокированном алгоритме случайного доступа с ортогональными преамбулами

А. А. Бурков ${ }^{\mathrm{a}}$, ассистент, orcid.org/0000-0002-0920-585X

В. В. Шнеер ${ }^{\sigma}$, канд. физ.-мат. наук, доцент, orcid.org/0000-0001-6750-6995

А. М. Тюрликов ${ }^{\mathrm{a}}$, доктор техн. наук, профессор, orcid.org/0000-0001-7132-094X, turlikov@k36.org

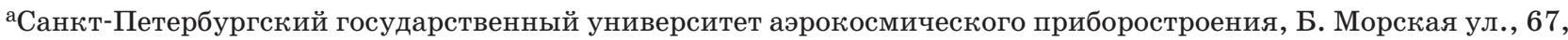
Санкт-Петербург, 190000, РФ

бШкола математических и компьютерных наук, Университет Хериота-Уатта, Эдинбург, EH14 4AS, Соединенное Королевство

Введение: в настоящее время разворачиваются первые версии сетей стандарта связи 5G и ведутся рассуждения о дальнейшем развитии сотовых сетей и переходе к стандарту 6G. Функционирование развивающейся идеи интернета вещей планируется в рамках сценария массовой межмашинной связи, к которому имеется ряд требований: очень высокая энергоэффективность, относительно невысокая задержка и достаточно надежная связь. Предполагается использование процедур случайного множественного доступа, так как ввиду особенностей трафика невозможно разработать политику разделения ресурсов канала. Для повышения эффективности случайного доступа можно применять класс неблокированных алгоритмов, использующих ортогональные преамбулы. Цель: расчет нижней границы средней задержки для класса неблокированных алгоритмов случайного множественного доступа с использованием ортогональных преамбул. Результаты: предложена модель системы с потенциально неограниченным числом абонентов, которые используют случайный неблокированный доступ для передачи данных по общему каналу связи с применением ортогональных преамбул. Для бесконечного числа преамбул доказано, что до интенсивности входного потока, равной 0,5671 , система работает стабильно. Получено замкнутое выражение для расчета средней задержки в стабильной системе в зависимости от интенсивности входного потока. Обосновано, что это выражение является нижней границей для средней задержки в системе с конечным числом преамбул. Для конечного числа преамбул выполнено имитационное моделирование. Результаты моделирования показали, что при увеличении числа преамбул интенсивность входного потока, при которой система работает стабильно, приближается к 0,5671, а средняя задержка стремится к нижней границе. При интенсивности входного потока, не превышающей 0,3 , достаточно порядка 100 ортогональных преамбул для достижения нижней границы. Практическая значимость: полученная граница дает возможность оценить снизу среднюю задержку в описанном классе алгоритмов. Ее использование позволяет определить возможность применения рассмотренного класса алгоритмов с точки зрения ограничений по средней задержке на стадии проектирования систем случайного множественного доступа.

Ключевые слова - случайный множественный доступ, предельная интенсивность входного потока, средняя задержка, преамбулы, mMTC, IoT

Для цитирования: Burkov A. A., Shneer S. V., Turlikov A. M. Lower bound for average delay in unblocked random access algorithm with orthogonal preambles. Информационно-управляющие систелы, 2020, № 3, с. 79-85. doi:10.31799/1684-8853-2020-3-79-85 For citation: Burkov A. A., Shneer S. V., Turlikov A. M. Lower bound for average delay in unblocked random access algorithm with orthogonal preambles. Informatsionno-upravliaiushchie sistemy [Information and Control Systems], 2020, no. 3, pp. 79-85. doi:10.31799/1684-8853-2020-3-79-85 